\title{
GROWTH PROBLEMS FOR A CLASS OF ENTIRE FUNCTIONS VIA SINGULAR INTEGRAL ESTIMATES
}

BY

\section{Daniel F. Shea ANd Stephen Wainger ${ }^{1}$}

Let $f$ be an entire function with zeros $\left\{z_{n}\right\}$, and let

$$
\begin{gathered}
M(r, f)=\max _{\theta}\left|f\left(r e^{i \theta}\right)\right|, \quad L(r, f)=\min _{\theta}\left|f\left(r e^{i \theta}\right)\right|, \\
n(r)=n(r, 0 ; f)=\sum_{\left|z_{n}\right| \leq r} 1, \\
\rho=\limsup _{r \rightarrow \infty} \frac{\log \log M(r, f)}{\log r} .
\end{gathered}
$$

We consider a problem motivated by a classical theorem of Pólya and Valiron; this gives lower bounds for

$$
c(f)=\limsup _{r \rightarrow \infty} \frac{n(r)}{\log M(r, f)}
$$

for functions of finite nonintegral order $\rho$. Let

$$
C(\rho)=\inf \{c(f): f \text { of order } \rho\} .
$$

Then Pólya [6] and Valiron [9] [10] proved, independently, that

$$
C(\rho)=\frac{1}{\pi} \sin \pi \rho \quad(0 \leq \rho \leq 1),
$$

$$
\frac{1}{\pi}|\sin \pi \rho| \geq C(\rho) \geq \frac{|\sin \pi \rho|}{A_{0}\{\log \rho+1\}|\sin \pi \rho|+\pi}(1<\rho<\infty),
$$

for an absolute constant $A_{0}$. The upper estimate in (3) comes from the Lindelöf functions

$$
f_{\rho}(z)=\prod_{n=1}^{\infty}\left(1+\frac{z}{n^{\sigma}}\right) \exp \left\{\sum_{k=1}^{q} \frac{1}{k}\left(\frac{z}{-n^{\sigma}}\right)^{k}\right\}\left(\sigma=\rho^{-1}, q=[\rho]\right),
$$

for which $n(r) \sim r^{\rho}, \log M(r, f) \sim \pi|\csc \pi \rho| r^{\rho}$ when $\rho$ is nonintegral and $r \rightarrow \infty$. We conjecture that these $f_{\rho}$, having all zeros regularly distributed on a single ray $\arg z=$ constant, are extremal for this problem, i.e. that $C(\rho)=$ $\pi^{-1}|\sin \pi \rho|$. But not even the order of magnitude of $C(\rho)$ is known, for $\rho$ large.

Received January 30, 1979.

${ }^{1}$ This research was supported in part by grants from the National Science Foundation. 
In this direction, we prove the existence of an absolute constant $A$ such that

$$
c(f) \geq A|\sin \pi \rho| \quad(1<\rho<\infty)
$$

for $f$ of order $\rho$, all of whose zeros lie on a single ray $\arg z=\pi$, say. For these $f$ our proof gives, in fact, an estimate for $n(r)$ in terms of

$$
B(r, f)=\sup _{|\theta|<\pi}\left|\log f\left(r e^{i \theta}\right)\right|
$$

for a fixed branch of $\log f(z)$.

THEOREM 1. Let $f$ have all zeros real and negative. Then

$$
\limsup _{r \rightarrow \infty} \frac{n(r)}{B(r, f)} \geq A|\sin \pi \rho| \quad(1<\rho<\infty) .
$$

We deduce (6) from a known Stieltjes transform representation for $\log f(z)$, cf. (1.2) below, by using singular integral estimates to obtain the $L^{p}$ norm inequality

$$
\left\{\int_{R_{n}}^{\infty}\left(\frac{B(r)}{r^{q+1}}\right)^{p} d r\right\}^{1 / p} \leq\left(A \sin \left(\frac{\pi}{p}\right)\right)^{-1}\left\{\int_{R_{n}}^{\infty}\left(\frac{n(r)}{r^{q+1}}\right)^{p} d r\right\}^{1 / p} .
$$

This is valid for $q=[\rho], p=(q+1-\rho)^{-1}+\varepsilon_{n}$ and suitable sequences $\varepsilon_{n} \rightarrow 0$, $R_{n} \rightarrow \infty$.

By iterating (7) we deduce the following:

Corollary. Let f have finite nonintegral order $\rho$. If all the zeros of flie on $v$ rays through 0 , then

$$
\begin{gathered}
c(f) \geq \frac{A}{v}|\sin \pi \rho|, \\
\limsup _{r \rightarrow \infty} \frac{\log L(r, f)}{n(r)} \geq-\frac{v}{A}|\csc \pi \rho|
\end{gathered}
$$

where $A$ is the constant in (6).

While (4) may remain true for all entire functions of order $\rho$, some restriction on the arguments of the zeros is essential in (6) and (9). W. K. Hayman has constructed entire $f$ of any order $\rho\left(\rho_{0} \leq \rho<\infty\right)$ with

$$
\limsup _{r \rightarrow \infty} \frac{\log L(r, f)}{n(r)} \leq-B_{0} \log \rho
$$

where $B_{0}$ and $\rho_{0}$ are positive absolute constants (cf. the estimates on pp. 501-503 of [4]).

Concerning the correct value of $C(\rho)$, Valiron [10] asserted that the lower estimate in (3) has the correct order of magnitude for large $\rho$, i.e. that

$$
c(f)<B_{1} / \log \rho
$$


for some $f$ of arbitrarily large half-integral order, but his argument is incorrect. Wahlund [11] claimed to have an example showing that $C(\rho)<\pi^{-1}|\sin \pi \rho|$ for nonintegral order $\rho>1$ but, as far as we know, this example was never published.

From (8) we see that functions which satisfy (10), with $\rho$ large and halfintegral, say, would have to have zeros with fairly complicated sets of arguments. However, these zeros could not be expected to be very uniformly spread throughout the plane, as in Hayman's examples [4], since those $f$ satisfy $n(r)>A_{1} \log M(r, f)\left(r_{0}<r<\infty\right)$ with $A_{1}$ independent of $\rho$.

The Pólya-Valiron result (3) was established at a special sequence of $r$-values (these days often called the Pólya peaks of $n(r)$ ), i.e. their proofs give

$$
\liminf _{j \rightarrow \infty} \frac{n\left(r_{j}\right)}{\log M\left(r_{j}, f\right)} \geq \frac{|\sin \pi \rho|}{A_{0}\{\log \rho+1\}|\sin \pi \rho|+\pi}
$$

for any entire $f$ of order $\rho$ and $\left\{r_{j}\right\}$ any sequence satisfying $r_{j} \rightarrow \infty$ and

$$
n(t) \leq n\left(r_{j}\right)\left(\frac{t}{r_{j}}\right)^{\rho-\varepsilon_{j}}\left(1 \leq t \leq r_{j}\right), \quad n(t) \leq n\left(r_{j}\right)\left(\frac{t}{r_{j}}\right)^{\rho+\varepsilon_{j}}\left(t \geq r_{j}\right)
$$

for some $\varepsilon_{j} \rightarrow 0(j \rightarrow \infty)$.

Perhaps surprisingly, the bound in (11) does have the correct order of magnitude, as an inequality at the Pólya peaks of $n(r)$, even for the functions of Theorem 1: In Section 3 we construct $f$, of arbitrarily large order $\rho$ and with negative zeros only, for which

$$
\limsup _{j \rightarrow \infty} \frac{n\left(r_{j}\right)}{\log M\left(r_{j}, f\right)}<\frac{2}{\log \rho}
$$

holds for every sequence $\left\{r_{j}\right\}$ of peaks of $n(r)$ (or of $\log M(r, f)$ ). Since these $f$ satisfy both (4) and (13), and since the second part of (3) is derived from (11), there is no reason to believe that (3) gives a good lower estimate for $C(\rho)$.

We mention that our inequalities (6)-(9) have restatements with $n(r)$ replaced by $N(r)=\int_{0}^{r} n(t) d t / t$, provided the constant $A$ is replaced throughout by $A / \rho$.

\section{Proof of Theorem 1}

We put $q=[\rho]$ and assume $f(0)=1$. Then for any $s>0$ we can write

$$
\log f(z)=\log \Pi_{s}(z)+R_{s}(z)
$$

where

$$
\begin{gathered}
\Pi_{s}(z)=\prod_{t_{n}>s}\left(1+\frac{z}{t_{n}}\right) \exp \left\{\sum_{k=1}^{q} \frac{1}{k}\left(\frac{z}{-t_{n}}\right)^{k}\right\} \quad\left(z_{n}=-t_{n}<0\right), \\
\left|R_{s}(z)\right| \leq C\left(\frac{r}{s}\right)^{q} \log M(2 s, f) \quad(|z|=r \geq 2 s)
\end{gathered}
$$


for a constant $C$ (Edrei and Fuchs [1], pp. 296, 313, 314).

After an integration by parts,

$$
\log \Pi_{s}(z)=(-1)^{q} z^{q+1} \int_{0}^{\infty} \frac{n\left(t, 0 ; \Pi_{s}\right)}{t^{q+1}} \frac{d t}{t+z} \quad(|\arg z|<\pi) .
$$

From (1.2) and (5),

$$
\begin{gathered}
B\left(r, \Pi_{s}\right)=r^{q+1} \sup _{\theta}\left|\int_{0}^{\infty} \frac{n\left(t, 0 ; \Pi_{s}\right)}{t^{q+1}} K(r, t, \theta) d t\right| \quad(0<r<\infty), \\
K(r, t, \theta)=e^{-i \theta} \frac{r+t \cos \theta+i t \sin \theta}{r^{2}+t^{2}+2 t r \cos \theta}
\end{gathered}
$$

so that $B\left(r, \Pi_{s}\right) / r^{q+1}$ can be considered a kind of maximal function. We shall establish the norm estimate

$$
\begin{gathered}
\int_{0}^{\infty}\left(\frac{B\left(r, \Pi_{s}\right)}{r^{q+1}}\right)^{p} d r \leq \alpha_{p} \int_{0}^{\infty}\left(\frac{n\left(r, 0 ; \Pi_{s}\right)}{r^{q+1}}\right)^{p} d r\left(p>\frac{1}{q+1-p}\right), \\
\alpha_{p}=\left(A \sin \left(\frac{\pi}{p}\right)\right)^{-p} .
\end{gathered}
$$

Assuming (1.4), we complete the proof of Theorem 1. Recall from (1.1) that $n\left(r, 0, \Pi_{s}\right)=0$ for $t \leq s$. Thus from (1.1), (1.4) and Minkowski's inequality, we obtain

$$
\begin{aligned}
\left\{\left.\int_{2 s}^{\infty}\left(\frac{B(r, f)}{r^{q+1}}\right)^{p} d r\right|^{1 / p} \leq\right. & \left\{\alpha_{p} \int_{0}^{\infty}\left(\frac{n\left(r, 0 ; \Pi_{s}\right)}{r^{q+1}}\right)^{p} d r\right\}^{1 / p} \\
& +\left(\frac{C \log M(2 s, f)}{s^{q}}\right)\left\{\int_{2 s}^{\infty} \frac{d r}{r^{p}}\right\}^{1 / p} \\
\leq & \left\{\alpha_{p} \int_{s}^{\infty}\left(\frac{n(r)}{r^{q+1}}\right)^{p} d r\right\}^{1 / p}+B_{1}(p) \frac{\log M(2 s, f)}{s^{q+1-1 / p}}
\end{aligned}
$$

with $B_{1}(p)=C(p-1)^{-1 / p}$. Since by Jensen's theorem $n(2 s)<2 \log M(4 s, f)$, we have

$$
\left\{\left.\int_{2 s}^{\infty}\left(\frac{B(r, f)}{r^{q+1}}\right)^{p} d r\right|^{1 / p} \leq\left\{\left.\alpha_{p} \int_{2 s}^{\infty}\left(\frac{n(r)}{r^{q+1}}\right)^{p} d r\right|^{1 / p}+B_{2}(p) \frac{\log M(4 s, f)}{s^{q+1-1 / p}}\right.\right.
$$

where $B_{2}(p)=B_{1}(p)+2 \alpha_{p}^{1 / p}$ is bounded for $p$ bounded away from 1 and $\infty$.

By an elementary argument [7, p. 208], there exist $x_{n} \rightarrow \infty, K_{n} \rightarrow \infty$ such that

$$
\log M(r, f)>\frac{1}{2} \log M\left(x_{n}, f\right)\left(\frac{r}{x_{n}}\right)^{\rho} \quad\left(x_{n} \leq r \leq K_{n} x_{n}\right) .
$$


Choose $s=s_{n}=x_{n} / 4$, so that for $\varepsilon>0$ and $p=(q+1-\rho)^{-1}(1+\varepsilon)$ we have, by (5),

$$
\begin{aligned}
\int_{2 s_{n}}^{\infty}\left(\frac{B(r, f)}{r^{q+1}}\right)^{p} d r & >\int_{x_{n}}^{K_{n} x_{n}}\left(\frac{\log M(r, f)}{r^{q+1}}\right)^{p} d r \\
& >\left(\frac{1-K_{n}^{-\varepsilon}}{\varepsilon}\right)\left(\frac{\log M\left(x_{n}, f\right)}{2 x_{n}^{q+1-1 / p}}\right)^{p} .
\end{aligned}
$$

Since we can choose $\varepsilon$ arbitrarily small, (1.6) implies

$$
(1-\eta) \int_{2 s_{n}}^{\infty}\left(\frac{B(r, f)}{r^{q+1}}\right)^{p} d r \leq \alpha_{p} \int_{2 s_{n}}^{\infty}\left(\frac{n(r)}{r^{q+1}}\right)^{p} d r
$$

where $\eta=\eta(\varepsilon) \rightarrow 0$ and $p \rightarrow(q+1-\rho)^{-1}$ when $\varepsilon \rightarrow 0$. Thus (1.5) yields (6) and (7).

To deduce the corollary, we write $f(z)=\prod_{j=1}^{v} f_{j}\left(e^{i \theta_{j}} z\right)$ where each $f_{j}(z)$ has negative zeros only, and genus $q$, and let $n_{j}(r)=n\left(r, 0 ; f_{j}\right)$,

Then

$$
\sigma(r)=\sum_{j=1}^{v} \log M\left(r, f_{j}\right), \quad \Sigma(r)=\sum_{j=1}^{v} B\left(r, f_{j}\right) .
$$

$$
\sigma(r) \leq \Sigma(r), \quad|\log | f\left(r e^{i \theta}\right) \| \leq \Sigma(r),
$$

and by (1.6) and the Minkowski, Jensen and Hölder inequalities,

$$
\begin{aligned}
\left\{\left.\int_{2 s}^{\infty}\left(\frac{\Sigma(r)}{r^{q+1}}\right)^{p} d r\right|^{1 / p}\right. & \leq \sum_{j=1}^{v}\left\{\alpha_{p} \int_{2 s}^{\infty}\left(\frac{n_{j}(r)}{r^{q+1}}\right)^{p} d r+B_{2}(p)^{p} \frac{\left(\log M\left(4 s, f_{j}\right)\right)^{p}}{s^{p(q+1)-1}}\right\}^{1 / p} \\
& \leq v^{1-1 / p} \alpha_{p}^{1 / p}\left|\int_{2 s}^{\infty}\left(\frac{n(r)}{r^{q+1}}\right)^{p} d r\right|^{1 / p}+B_{2}(p) \frac{\sigma(4 s)}{s^{q+1-1 / p}}
\end{aligned}
$$

Choose $x_{n}, K_{n}$ to satisfy (1.7) with $\log M(r, f)$ replaced by $\sigma(r)$. Using (1.11) and the first inequality in (1.9), the argument used in (1.8) yields

$$
\begin{aligned}
\frac{\sigma\left(4 s_{n}\right)}{s_{n}^{q+1-1 / p}} & \leq \varepsilon^{1 / p} C B_{2}(p)\left\{\left.\int_{2 s_{n}}^{\infty}\left(\frac{\sigma(r)}{r^{q+1}}\right)^{p} d r\right|^{1 / p}\right. \\
& \leq \varepsilon^{1 / p} B_{3}(p)\left\{\left.\int_{2 s_{n}}^{\infty}\left(\frac{n(r)}{r^{q+1}}\right)^{p} d r\right|^{1 / p}\right.
\end{aligned}
$$

where as before $p=(q+1-\rho)^{-1}(1+\varepsilon)$. Thus, (1.11) and the second inequality in (1.10) imply

$$
(1-\eta) \int_{2 s_{n}}^{\infty}\left(\sup _{\theta} \frac{|\log | f\left(r e^{i \theta}\right) \|}{r^{q+1}}\right)^{p} d r \leq v^{p-1} \alpha_{p} \int_{2 s_{n}}^{\infty}\left(\frac{n(r)}{r^{q+1}}\right)^{p} d r
$$

where $\eta$ and $p$ behave as in (1.9). We deduce (8) and (9), in a stronger form than stated: In those inequalities, $v$ can be replaced by $v^{\rho-q}$. 


\section{Proof of (1.4)}

For fixed $s>0$, let

$$
\phi(r)=n\left(r, 0 ; \Pi_{s}\right) r^{-q-1}, \quad \Phi(r)=B\left(r, \Pi_{s}\right) r^{-q-1},
$$

so that (1.3) becomes

$$
\Phi(r)=\sup _{0<\theta<\pi}\left|\int_{0}^{\infty} \phi(t) K(r, t, \theta) d t\right| .
$$

We proceed to estimate

$$
\|\Phi\|_{p}=\left\{\int_{0}^{\infty} \Phi(r)^{p} d r\right\}^{1 / p}
$$

in terms of the Hardy-Littlewood maximal function

$$
M \phi(r)=\sup _{\varepsilon>0} \frac{1}{2 \varepsilon} \int_{|t-r|<\varepsilon} \phi(t) d t
$$

and the maximal Hilbert transform

We shall prove

$$
H^{*} \phi(r)=\sup _{\varepsilon>0}\left|\int_{|t-r|>\varepsilon} \frac{\phi(t)}{t-r} d t\right| .
$$

$$
\Phi(r) \leq 12 M \phi(r)+H^{*} \phi(r)+10 \int_{0}^{\infty} \frac{\phi(t)}{t+r} d t .
$$

Assuming (2.1), we deduce

$$
\|\phi\|_{p} \leq 12\|M \phi\|_{p}+\left\|H^{*} \phi\right\|_{p}+A(p)\|\phi\|_{p}
$$

for $(q+1-\rho)^{-1}<p<\infty$; here $A(p)=10 \pi \csc (\pi / p)$ by a simple application of Minkowski's inequality (cf. [8, p. 271]).

By a classical estimate (e.g. [8, p. 7]),

$$
\|M \phi\|_{p} \leq \frac{10 p}{p-1}\|\phi\|_{p} \quad\left(p>(q+1-\rho)^{-1}\right) .
$$

We also have

$$
\left\|H^{*} \phi\right\|_{p} \leq A_{2}\left(\frac{p}{p-1}+p\right)\|\phi\|_{p} \quad\left(p>(q+1-\rho)^{-1}\right)
$$

for an absolute constant $A_{2}$; this can be derived for example when $p \geq 2$ from the estimate of $[8, p .67]$ together with (2.3), and for $1<p<2$ from [8, pp. 42, $21,22]$. (Or, compare the estimates in [12, pp. 256-258] for an equivalent [15, p. 256] problem.) Since

$$
p \leq \frac{p}{p-1} \leq \pi \csc \left(\frac{\pi}{p}\right)(1<p \leq 2), \quad \frac{p}{p-1} \leq p \leq \pi \csc \left(\frac{\pi}{p}\right)(p \geq 2),
$$

(2.1)-(2.4) yield (1.4). 
It remains to prove (2.1). We have from (1.3) that

$$
\Phi(r) \leq \sum_{j=1}^{3}\left(\sup _{0<\theta<\pi}\left|\int_{0}^{\infty} \phi(t) K_{j}(r, t, \pi-\theta) d t\right|\right)
$$

where, if we let $D=(r-t)^{2}+2 \operatorname{tr}(1-\cos \theta)$,

$$
K_{1}=\frac{r-t}{D}, \quad K_{2}=\frac{t(1-\cos \theta)}{D}, \quad K_{3}=\frac{t \sin \theta}{D} .
$$

Now $K_{2}<4 /(r+t)$ and, if we let $\varepsilon^{2}=2 r^{2}(1-\cos \theta)$, then

$$
K_{1}=\frac{r-t}{(r-t)^{2}+\varepsilon^{2}}+\Delta_{1}=Q+\Delta_{1}
$$

where $0 \leq \Delta_{1} \leq 2 /(r+t)$. We estimate $\int_{0}^{\infty} \phi Q$ in terms of $H^{*} \phi, M \phi$ and

$$
\phi * P_{\varepsilon}(r)=\int_{0}^{\infty} \phi(t) P_{\varepsilon}(r-t) d t, \quad P_{\varepsilon}(r)=\varepsilon\left(r^{2}+\varepsilon^{2}\right)^{-1},
$$

by observing that

$$
\sup _{\varepsilon>0}\left|\int_{0}^{\infty} \phi(t) Q d t\right| \leq \sup _{\varepsilon>0}\left|\int_{|t-r| \geq \varepsilon} \phi Q\right|+\sup _{\varepsilon>0}\left|\int_{|t-r|<\varepsilon} \phi Q\right|=T_{1}+T_{2}
$$

where, since $\phi \geq 0$ and $0<\theta<\pi$,

$$
T_{1} \leq H^{*} \phi(r)+2 \sup _{\varepsilon>0} \phi * P_{\varepsilon}(r), \quad T_{2} \leq 2 M \phi(r) .
$$

In $K_{3}$, set $\psi=\frac{1}{2} \theta$ and $\varepsilon=2 r \sin \psi$ so that

where

$$
K_{3} \leq \frac{2 t \sin \psi}{(r-t)^{2}+4 r t \sin ^{2} \psi}=P_{\varepsilon}(r-t)+\Delta
$$

Thus

$$
|\Delta| \leq \frac{2 \sin \psi|r-t|}{(r-t)^{2}+4 r^{2} \sin ^{2} \psi}
$$

$$
\sup _{0<\psi<\frac{1}{2} \pi}\left|\int_{0}^{\infty} \phi(t) \Delta d t\right| \leq \sup _{0<\varepsilon<2 r}\left\{\int_{|t-r|<\varepsilon}+\int_{|t-r| \geq \varepsilon}|\phi| \Delta \mid\right.
$$

where

$$
|\Delta| \leq \frac{1}{r} \leq \frac{4}{r+t} \quad(|t-r|<\varepsilon<2 r)
$$

and, since $\varepsilon=2 r \sin \psi$,

$$
\begin{aligned}
\int_{|t-r| \geq \varepsilon} \phi|\Delta| & \leq \frac{1}{2 r} \int_{0}^{(r-\varepsilon)^{+}} \phi+\frac{1}{2 r} \int_{r+\varepsilon}^{3 r} \phi+\int_{3 r}^{\infty} \phi(t) \frac{\varepsilon}{r(t-r)} d t \\
& \leq 4 \int_{|t-r| \geq \varepsilon} \frac{\phi(t)}{r+t} d t .
\end{aligned}
$$


Summing up,

$$
\Phi(r) \leq 3 \sup _{\varepsilon>0} \phi * P_{\varepsilon}(r)+2 M \phi(r)+H^{*} \phi(r)+10 \int_{0}^{\infty} \frac{\phi(t)}{r+t} d t .
$$

Now (2.1) follows from the fact [8, p. 62] that $\sup _{\varepsilon>0} \phi * P_{\varepsilon}(r) \leq \pi M \phi(r)$.

\section{An example}

As far as we know, earlier studies comparing the growth of $n(r)$ [or $N(r)$ ] with that of $\sup _{\theta}\left|f\left(r e^{i \theta}\right)\right|$ have always, explicitly or implicitly, computed this growth at the Pólya peaks of $n(r)$ [or $N(r)]$. This is true of the papers of Valiron [9], Pólya [6] and Wahlund [11], as well as of more recent papers of Williamson [14] and Fuchs [2]. We give an example showing that the order of magnitude in $\rho$ of the classical estimate (11) cannot be improved at the peaks (of $n(r), N(r)$ or $\log M(r, f))$.

This example is related to a question raised by Williamson in [14]. The method of [14] gives a sharp estimate

$$
\log M\left(r_{j}, f\right) \leq\{\pi \rho /|\sin \pi \rho|+o(1)\} N\left(r_{j}\right) \quad(j \rightarrow \infty)
$$

at peaks $\left\{r_{j}\right\}$ of $N(r)$, when $f$ has negative zeros only and satisfies Williamson's additional assumption $A[14, \mathrm{p} .500]$ on the location of the points $z$ where $|f(z)|=M(|z|, f)$. In [13], Wheeler shows that $A$ does not hold for all $f$ with negative zeros. This fact also follows from our examples (3.5) below, since (3.1) itself fails for these $f$.

In [2], Fuchs proves an inequality implying

$$
\begin{aligned}
\sup _{\theta} \log \mid f\left(r_{j} e^{i(\theta+\beta)}\right) & f\left(r_{j} e^{i(\theta-\beta)}\right) \mid \\
& \leq\{2 \pi \rho /|\sin \pi \rho|+o(1)\} N\left(r_{j}\right) \quad(j \rightarrow \infty)
\end{aligned}
$$

for certain $\beta$ near $\pi / 2 \rho$ and $\left\{r_{j}\right\}$ any sequence of peaks of $N(r)$. Our estimates (3.7)-(3.8) for the functions (3.5) show that one cannot expect (3.2) to remain valid for $\beta$ near 0. (Compare also [3], where Fuchs proves a version of (3.2) not uniform in $\theta$, but for which the restriction on $\beta$ is removed.)

Our examples are defined as follows: Let $\rho>4$ be given and let $\left\{b_{k}\right\}$ be a sequence with $0<b_{k}<b_{k+1}(k \geq 1)$, and $b_{k} \rightarrow \infty$ so that

$$
\sum_{j=1}^{k-1} b_{j}^{\rho}=o\left(b_{k}^{1 / 2}\right) \quad(k \rightarrow \infty) \text {. }
$$

Let $q=[\rho]$ and define $v(t)=0\left(0 \leq t \leq b_{1}\right), b_{0}=0$ and

$$
v(t)= \begin{cases}v\left(2 b_{k-1}\right)+\left(t-b_{k}\right) t^{\rho-1} & \text { if } b_{k} \leq t \leq 2 b_{k}, k \geq 1 \\ v\left(2 b_{k}\right) & \text { if } 2 b_{k} \leq t<b_{k+1}, k \geq 1 .\end{cases}
$$

We can now define

$$
f(z)=\prod_{n=1}^{\infty} E\left(\frac{z}{-a_{n}}, q\right)
$$


where $E(z, q)=(1-z) \exp \left(\sum_{k=1}^{q} z^{k} / k\right)$ and the sequence $\left\{a_{n}\right\}$ is determined by $0<a_{n}<a_{n+1}(n \geq 1)$ and $n(t)=n(t, 0 ; f)=[v(t)]$. Let $r_{k}=2 b_{k}(k \geq 1)$. Then $\left\{r_{k}\right\}$ is a sequence of peaks of $n(t)$, as defined in (12). Further, a little calculation shows that any sequence $\left\{R_{k}\right\}$ of peaks of $n(t)$ must satisfy

$$
1 \leq r_{k}^{\prime} / R_{k} \leq 1+o(1) \quad(k \rightarrow \infty)
$$

for some subsequence $\left\{r_{k}^{\prime}\right\}$ of $\left\{r_{k}\right\}$, say $\left\{r_{k}^{\prime}\right\}=\left\{r_{k}\right\}$. We shall prove

$$
\lim \inf \log M\left(R_{k}, f\right) / n\left(R_{k}\right)>2^{-1 / 2} \log \rho-3 .
$$

Thus, for $\rho$ sufficiently large, these $f$ satisfy (13). Further, since (3.4) and (3.6) imply

$$
n(r) / N(r) \rightarrow A(\rho)
$$

for $r$ near $R_{k}$ and $k \rightarrow \infty$, with $A(\rho)>2 \rho$ for $\rho_{0}<\rho<\infty$, it is clear that these $f$ fail to satisfy (3.1). Also, if $\left\{t_{k}\right\}$ is any sequence of peaks of $\log M(r, f)$, it is easy to see, from (3.4) and (12), that (13) remains valid with $r_{k}$ replaced by $t_{k}$.

To prove (3.7), let $|z|=R_{k}$ and write

$$
\log |f(z)|=\int_{b_{k}}^{r_{k}} \log \left|E\left(\frac{z}{-t}, q\right)\right| d n(t)+S_{k}(z)
$$

where, by (3.3), $S_{k}(z) / n\left(R_{k}\right) \rightarrow 0(k \rightarrow \infty)$. Writing $R_{k}=R, r_{k}=r$ and $z=R e^{i \theta}$,

$$
\begin{aligned}
\log |f(z)|=n(r) \log \left|E\left(\frac{z}{-r}, q\right)\right| & +(-1)^{q} \int_{b_{k}}^{r} n(t)\left(\frac{r}{t}\right)^{q+1} \\
& \times \frac{r \cos q \theta+t \cos (q+1) \theta}{r^{2}+t^{2}+2 t r \cos \theta} d t-o(n(r))
\end{aligned}
$$

when $k \rightarrow \infty$, uniformly in $\theta$. We now put $\theta=\pi-\pi / 4 q$ and $t=R s$ in (3.9), and obtain

$$
\begin{aligned}
\liminf _{k \rightarrow \infty} \frac{\log M\left(R_{k}, f\right)}{n\left(R_{k}\right)} & \\
& \geq \cos \frac{\pi}{4} \int_{1 / 2}^{1} \frac{2 s-1}{s^{q+1-\rho}} \frac{1-s}{(1-s)^{2}+q^{-2}} d s+\log \left|E\left(e^{\pi i / 4 q}, q\right)\right| .
\end{aligned}
$$

Here the integral is bounded below by

$$
\int_{1 / 2}^{1}(2 s-1) \frac{1-s}{(1-s)^{2}+q^{-2}} d s=2 \int_{1 / 2}^{1}\left\{\int_{0}^{1-s} \frac{x d x}{x^{2}+q^{-2}}\right\} d s>\log \left(\frac{q}{2}\right)-1 .
$$

Since $\log \left|E\left(e^{\pi i / 4 q}, q\right)\right|>-1$, (3.7) follows at once. 


\section{REFERENCES}

1. A. EdReI and W. H. J. Fuchs, On the growth of meromorphic functions with several deficient values, Trans. Amer. Math. Soc., vol. 93 (1959), pp. 292-328.

2. W. H. J. FucHS, An inequality involving the absolute value of an entire function and the counting function of its zeros, Comment. Math. Helv., vol. 53 (1978), pp. 135-141.

3. W. H. J. Fuchs, On the growth of meromorphic functions on rays, preprint.

4. W. K. Hayman, The minimum modulus of large integral functions, Proc. London Math. Soc. (3), vol. 2 (1952), pp. 469-512.

5. - Meromorphic functions, Oxford Univ. Press, New York, 1964.

6. G. Pólya, Bemerkungen über unendlichen Folge und ganzen Funktionen, Math. Ann., vol. 88 (1923), pp. 169-183.

7. D. F. SHEA, On the Valiron deficiencies of meromorphic functions of finite order, Trans. Amer. Math. Soc., vol. 124 (1966), pp. 201-227.

8. E. M. SteIN, Singular integrals and differentiability properties of functions, Princeton Univ. Press, Princeton, N.J., 1970.

9. G. VAliron, Sur les fonctions entrières d'ordre fini et d'ordre nul, et en particulier les fonctions $\dot{a}$ correspondence régulière, Ann. Fac. Sci. Univ. Toulouse (3), vol. 5 (1913), pp. 117-257.

10. - A propos d'un mémoire de M. Pólya, Bull. Sci. Math. (2), vol. 48 (1924), pp. 9-12.

11. A. WAHLUND, Uber einen Zusammenhang zwischen dem Maximalbetrage der ganzen Funktionen und seiner unteren Grenze nach dem Jensen'schen Theoreme, Arkiv. for Math. 21A, Nr. 23 (1929).

12. R. L. Wheeden and A. Zygmund, Measure and Integral, Marcel Dekker, New York, 1977.

13. R. L. WHEELER, A maximum modulus analogue of Valiron's tauberian theorem for entire functions, Quart. J. Math. (Oxford) (2), vol. 24 (1973), pp. 315-331.

14. J. Williamson, Remarks on the maximum modulus of an entire function with negative zeros, Quart. J. Math. (Oxford) (2), vol. 21 (1970), pp. 497-512.

15. A. Zygmund, Trigonometric Series (2nd edition), vol. 2, Cambridge University Press, Cambridge, 1959.

UNIVERSITY OF WISCONSIN

Madison, Wisconsin 